\title{
A Group Construction Method with Applications to Deriving Pruned Interconnection Networks
}

\author{
Wenjun Xiao and Behrooz Parhami, Fellow, IEEE
}

\begin{abstract}
A number of low degree and, thus, low complexity, Cayley-graph interconnection structures, such as honeycomb and diamond networks, are known to be derivable by systematic pruning of $2 \mathrm{D}$ or 3D tori. In this paper, we extend these known pruning schemes via a general algebraic construction based on commutative groups. We show that, under certain conditions, Cayley graphs based on the constructed groups are pruned networks when Cayley graphs of the original commutative groups are $k \mathrm{D}$ tori. Thus, our results offer a general mathematical framework for synthesizing and exploring pruned interconnection networks that offer lower node degrees and, thus, smaller VLSI layout and simpler physical packaging. Our constructions also lead to new insights, as well as new concrete results, for previously known interconnection schemes such as honeycomb and diamond networks.
\end{abstract}

Index Terms-Algebraic structure, Cayley graph, distributed system, geometric group theory, interconnection network, network diameter, parallel processor architecture, pruning scheme, VLSI realization.

\section{INTRODUCTION}

$\mathrm{T}$ HE fact that Cayley (di)graphs and coset graphs are excellent models for interconnection networks, studied in connection with parallel processing and distributed computation, is widely acknowledged [1], [2], [4]. Many well-known interconnection networks are Cayley (di)graphs or coset graphs. For example, hypercube, butterfly, and cube-connected cycles networks are Cayley (di)graphs, while de Bruijn and shuffle-exchange networks are Cayley coset graphs [4], [11]. Unfortunately, much of the extensive body of work on interconnection networks consists of ad hoc design and evaluation: proposing a new interconnection scheme and showing it to be superior to some previously studied network(s) with respect to one or more performance or complexity attributes. Whereas Cayley (di)graphs have been used to explain and unify interconnection networks with some success, much work remains to be done. Heydemann [4] believes that there is a lack of general theorems pertaining to Cayley (di)graphs and advocates the exploitation of more group theory to study their properties.

One approach to reducing the implementation complexity of a network and, hence, increasing its performance with constant cost is systematic pruning of links. Under certain conditions, when the original networks are Cayley graphs, so are the pruned versions [9], [10]. Thus, the pruned networks maintain node symmetry while benefiting from lower node degree, sparser wiring, and simpler layout. For example, in one type of construction based on 2D or

- W.J. Xiao is with the Department of Computer Science, South China University of Technology, Guangzhou 510641, P.R. China.

E-mail:wjxiao@scut.edu.cn.

- B. Parhami is with the Department of Electrical and Computer Engineering, University of California, Santa Barbara, Santa Barbara, CA 93106-9560. E-mail: parhami@ece.ucsb.edu.

Manuscript received 14 July 2005; revised 13 Feb. 2006; accepted 5 Apr. 2006; published online 5 Jan. 2007.

Recommended for acceptance by Y. Pan.

For information on obtaining reprints of this article, please send e-mail to: tpds@computer.org, and reference IEEECS Log Number TPDS-0336-0705. Digital Object Identifier no. 10.1109/TPDS.2007.1002.

$1045-9219 / 07 / \$ 25.00$ C 2007 IEEE
3D torus (a Cayley graph of a finite commutative group $G$ ), defining a new group operator $\otimes$ on $G$ leads to the group $(G, \otimes)$ and, thereby, a Cayley-graph formulation of the pruned network. Certain known interconnection architectures, such as cube-connected cycles, honeycomb, and diamond networks, may be constructed in this manner.

In this paper, we propose a general method for deriving new groups from a finite commutative group $G$. Our method generalizes previously proposed pruning schemes and establishes a systematic and uniform framework for constructing new networks. Armed with this method, we also obtain new results on previously proposed networks such as diamond and honeycomb networks, the latter of which has important applications in wireless communication [7], [11]. Before proceeding further, we introduce key definitions and notations related to interconnection networks and (di)graphs, Cayley (di)graphs, in particular. For more definitions and basic results on graphs and groups, we refer the reader to [3], for instance, and, for interconnection network concepts, to [6], [8]. A list of key notation for this paper is presented in Table 1 for ease of reference. Unless noted otherwise, all graphs in this paper are undirected.

A digraph $\Gamma=(V, E)$ is defined by a set $V$ of vertices and a set $E$ of arcs or directed edges. The set $E$ is a subset of elements $(u, v)$ of $V \times V$. If the subset $E$ is symmetric, that is, $(u, v) \in E$ implies $(v, u) \in E$, we identify two opposite arcs $(u, v)$ and $(v, u)$ by the undirected edge $(u, v)$. Because we deal primarily with undirected graphs in this paper, no problem arises from using the same notation $(u, v)$ for a directed arc from $u$ to $v$ or an undirected edge between $u$ and $v$.

Let $G$ be a finite group and $S$ a subset of $G$. The subset $S$ is said to be a generating set for $G$ if every element of $G$ can be expressed as a finite product of the powers of its elements, called the generators of $G$. We also say that $G$ is generated by $S$. The Cayley digraph of the group $G$ and the subset $S$, denoted by $\operatorname{Cay}(G, S)$, has vertices that are 
TABLE 1

List of Key Notation for This Paper

\begin{tabular}{|c|c|}
\hline$\bullet \leq \bullet$ & Subgroup relationship \\
\hline$\%$ & Set of (right) cosets \\
\hline$\bullet \times \bullet$ & Graph or set cross-product \\
\hline$\bullet^{t}$ & The vector "•" transposed \\
\hline$\bullet^{(i)}$ & The symbol "•" repeated $i$ times \\
\hline$(\bullet, \bullet)$ & Edge \\
\hline$\langle\bullet\rangle$ & Cyclic group \\
\hline$\rightarrow$ & Mapping \\
\hline$\cong$ & Isomorphic to \\
\hline $\operatorname{Diam}(\bullet)$ & Diameter of a graph \\
\hline$\Gamma, \Delta, \Sigma$ & Graphs or digraphs \\
\hline$\phi$ & Homomorphism \\
\hline 4 & The identity element of a group \\
\hline$a, b, c, d$ & Unit vectors \\
\hline $\operatorname{Cay}(\bullet)$ & Cayley graph \\
\hline$C C C_{q}$ & Cube-connected cycles of order $q$ \\
\hline $\operatorname{Cos}(\bullet)$ & Coset graph \\
\hline $\operatorname{dist}(\bullet)$ & Distance function \\
\hline$E(\bullet)$ & Edge set of a graph \\
\hline$f(\bullet)$ & Pruning function \\
\hline$G, H$ & Groups \\
\hline$I_{q}$ & The identity matrix of order $q$ \\
\hline$K, N$ & Subgroups \\
\hline$M, Q$ & (Quasi-)permutation matrices \\
\hline$S, T$ & Generator sets, subsets of $G$ \\
\hline$V(\bullet)$ & Vertex set of a graph \\
\hline$x, y, z$ & Node labels (bit vectors) \\
\hline$Z_{k}$ & Cyclic group of order $k$ \\
\hline$Z_{k}^{q}$ & Elementary abelian group of order $k^{q}$ \\
\hline
\end{tabular}

elements of $G$ and arcs that are ordered pairs $(g, g s)$ for $g \in G, s \in S$. If $S$ is a generating set of $G$, then we say that $\operatorname{Cay}(G, S)$ is the Cayley digraph of $G$ generated by $S$. If $\pm \notin S$ ( 1 is the identity element of $G$ ) and $S=S^{-1}$, then $\operatorname{Cay}(G, S)$ is a simple graph.

Assume that $\Gamma$ and $\Sigma$ are two digraphs. The mapping $\phi$ of $V(\Gamma)$ to $V(\Sigma)$ is a homomorphism from $\Gamma$ to $\Sigma$ if, for any $(u, v) \in E(\Gamma)$, we have $(\phi(u), \phi(v)) \in E(\Sigma)$. In particular, if $\phi$ is a bijection such that both $\phi$ and the inverse of $\phi$ are homomorphisms, then $\phi$ is called an isomorphism of $\Gamma$ to $\Sigma$. Let $G$ be a finite group and $S$ a subset of $G$. Assume that $K$ is a subgroup of $G$ (denoted as $K \leq G$ ). Let $G / K$ denote the set of the right cosets of $K$ in $G$. The (right) coset graph of $G$ with respect to subgroup $K$ and subset $S$, denoted by $\operatorname{Cos}(G, K, S)$, is the digraph with vertex set $G / K$ such that there exists an arc $\left(K g, K g^{\prime}\right)$ if and only if there exists $s \in S$ and $K g s=K g^{\prime}$.

The remainder of our presentation is organized as follows: In Section 2, we introduce a number of pruned networks. In Section 3, we discuss a general method of constructing a new group from a given finite commutative group. In Section 4, we apply the aforementioned method to torus networks, thereby deriving a unified and systematic scheme for constructing new interconnection networks. In Section 5, we provide some new results on diamond and other networks. Section 6 contains our conclusions.

\section{Some Known Pruned Networks}

Assume that $q$ is an integer. Let $G=Z_{k_{1}} \times Z_{k_{2}} \times \cdots \times Z_{k_{q}}$, where $Z_{k_{i}}$ is a cyclic group of order $k_{i}$ and $k_{i}$ is an integer for $1 \leq i \leq q$. Thus, $G$ is a commutative group that is a direct product of the cyclic groups $Z_{k_{i}}$ for $1 \leq i \leq q$. Let $s_{i}=$ $\left(0^{(i-1)}, 1,0^{(q-i)}\right)$ be a vector of dimension $q$ for $1 \leq i \leq q$ and $S=\left\{ \pm s_{i} \mid 1 \leq i \leq q\right\}$. Suppose that $\Gamma=\operatorname{Cay}(G, S)$, that is, $\Gamma$ is a Cayley graph of the group $G$ and the generator set $S$. The network $\Gamma$ just defined is a $q$-dimensional (or $q \mathrm{D}$ ) torus. By pruning links of the graph $\Gamma$ in a unified and systematic fashion, we can obtain a pruned network which is still a Cayley graph. We give some known examples in the following:

Example 1. Cube-connected cycles $C C C_{q}$. Let $G=Z_{2}^{q} \times Z_{q}$. For any $x \in G$, we write $x=\left(x_{1}, x_{2}, \ldots, x_{q}, x_{q+1}\right)^{t}$, where $t$ denotes transpose. For $x, y \in G$, define a new group operator $\otimes$ as

$$
x \otimes y=x+\left[\begin{array}{cccccc}
0 & 0 & \cdots & 0 & 1 & 0 \\
1 & 0 & \cdots & 0 & 0 & 0 \\
0 & 1 & \cdots & 0 & 0 & 0 \\
\vdots & \vdots & \ddots & \vdots & \vdots & \vdots \\
0 & 0 & \cdots & 1 & 0 & 0 \\
0 & 0 & \cdots & 0 & 0 & 1
\end{array}\right]^{f(x)} y
$$

where the matrix is of order $q+1$ and $f$ is a function from $G$ to $Z_{q}$ such that $f(x)=-x_{q+1}$. It can be verified that $H=$ $(G, \otimes)$ is a new group under the group operator $\otimes$. Let $S$ be as above and $\Sigma=\operatorname{Cay}(H, T)$, where $T=\left\{s_{1}, s_{q+1}, s_{q+1}^{-1}\right\}$. Then, $\Sigma$ is known as cube-connected cycles $C C C_{q}$. It is the pruned network obtained from the graph $\Gamma=\operatorname{Cay}(G, S)$, which is a $(q+1) \mathrm{D}$ torus [10]. Fig. 1 depicts the $C C C_{4}$ network with $2^{4} \times 4=64$ nodes.

Example 2. Honeycomb network. Let $G=Z_{l} \times Z_{k}$ and $S=$ $\left\{ \pm s_{1}, \pm s_{2}\right\}$ as above, where $l$ and $k$ are even integers. Then, $\Gamma=\operatorname{Cay}(G, S)$ is a $2 \mathrm{D}$ torus. Define a group operator $\otimes$ for $x, y \in G$ as:

$$
x \otimes y=x+\left[\begin{array}{cc}
-1 & 0 \\
0 & 1
\end{array}\right]^{x_{1}+x_{2}} y .
$$

Let $H=(G, \otimes)$ and $T=\left\{s_{1}, s_{2}, s_{2}^{-1}\right\}$. Then, $\Sigma=\operatorname{Cay}(H, T)$ is a form of pruned 2D torus known as honeycomb network [9]. Fig. 2 depicts a 64-node honeycomb network with $l=k=8$.

Example 3. Diamond network. We only consider the 3D diamond network. Let $G=Z_{k}^{3}$. For $x, y \in G$, define a new group operator $\otimes$ as

$$
x \otimes y=x+\left[\begin{array}{ccc}
-1 & 0 & 0 \\
0 & -1 & 0 \\
0 & 0 & 1
\end{array}\right]^{f(x)} y
$$

where $f(x)=x_{1}+x_{2}+x_{3}$. Let $H=(G, \otimes), T=\left\{s_{1}, s_{2}, s_{3}\right.$, $\left.s_{3}^{-1}\right\}, \Sigma=\operatorname{Cay}(H, T)$. Then, it is known that $H$ is a new group under the group operator $\otimes$ and $\Sigma$ is the 3 D diamond network [9]. Fig. 3 depicts a 64-node diamond network with $k=4$. 


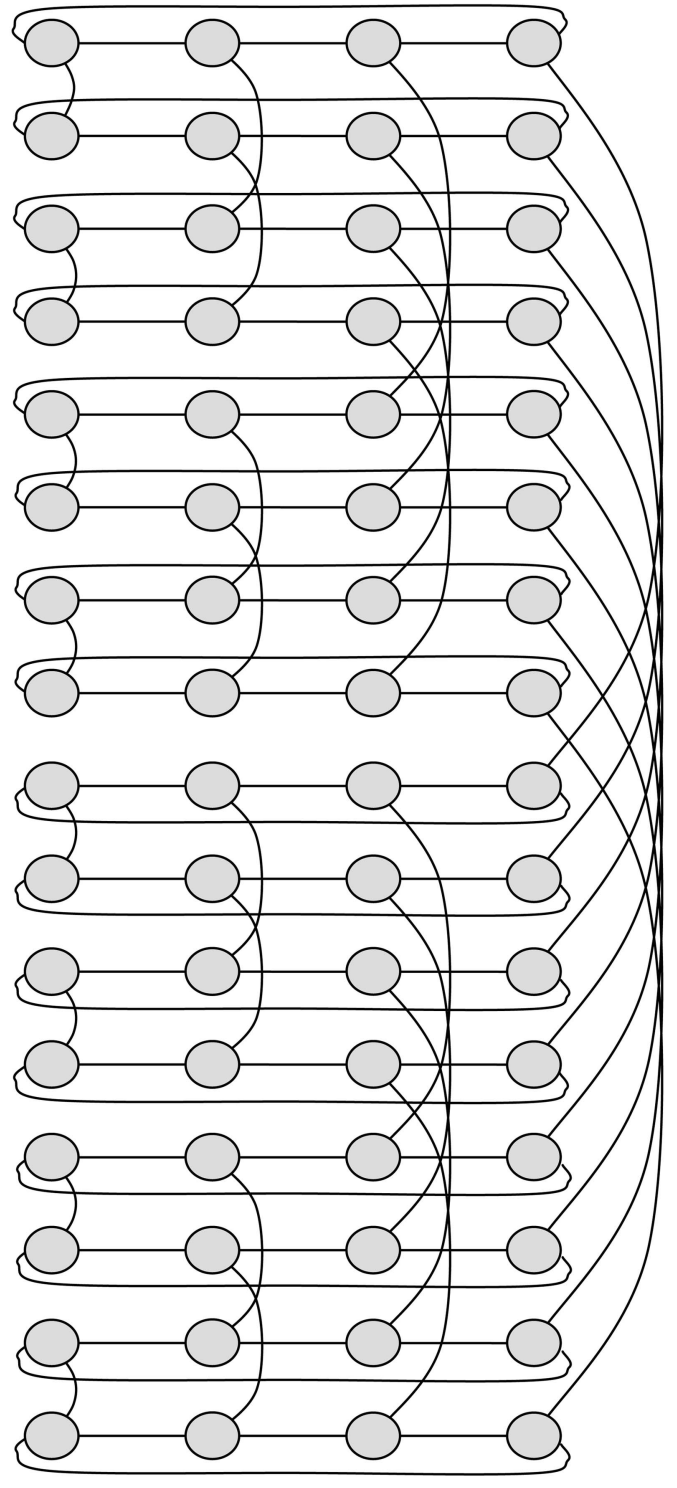

Fig. 1. The cube-connected cycles network $\mathrm{CCC}_{4}$.

\section{Group Construction from Commutative GROUPS}

In Section 2, we defined a new group operator $\otimes$ based on a given commutative group $G$ and obtained a new group $H=(G, \otimes)$. Let $\Gamma=\operatorname{Cay}(G, S)$ be the Cayley graph of the group $G$ and the generator set $S$. Then, the Cayley graph $\Sigma=\operatorname{Cay}(H, T)$ is a pruned network of the graph $\Gamma$, where $T \subseteq S$. For each of the examples in Section 2, a matrix $M$ appeared in the definition of $x \otimes y$. A natural question is: What conditions should the matrix $M$ satisfy for defining a new group operator $\otimes$ on the set $G$ ?

For the operator $\otimes$ on $G$ to define a group, it must satisfy the following three conditions:

1. There is an identity $t$ in $G$ such that $x \otimes t=x$ for any $x \in G$.

2. Any element $x$ of $G$ has a right inverse $x^{-1}$ in $G$ such that $x \otimes x^{-1}=1$.

3. For any three elements $x, y, z$ of $G$, the associative law $(x \otimes y) \otimes z=x \otimes(y \otimes z)$ holds.

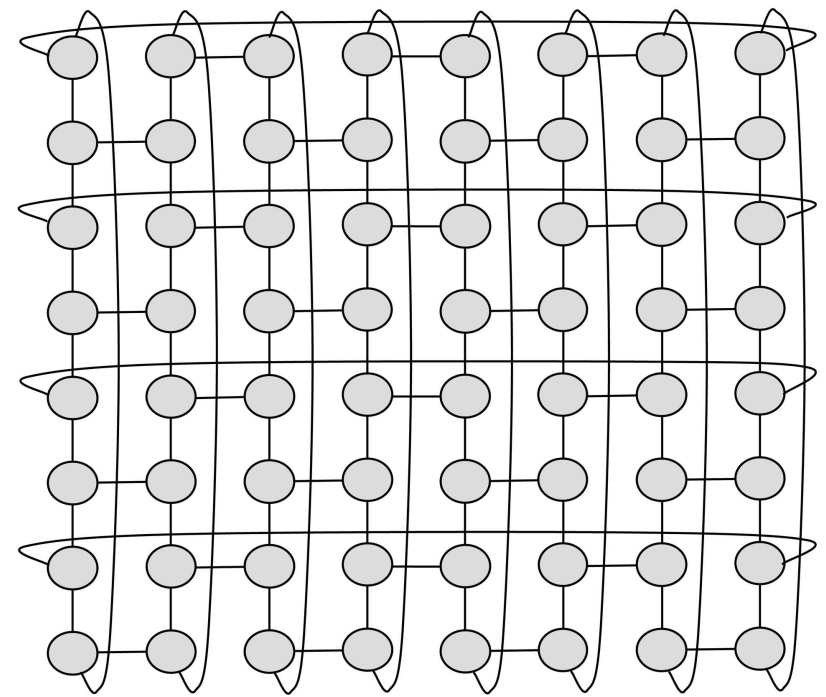

Fig. 2. A 2D honeycomb torus network.

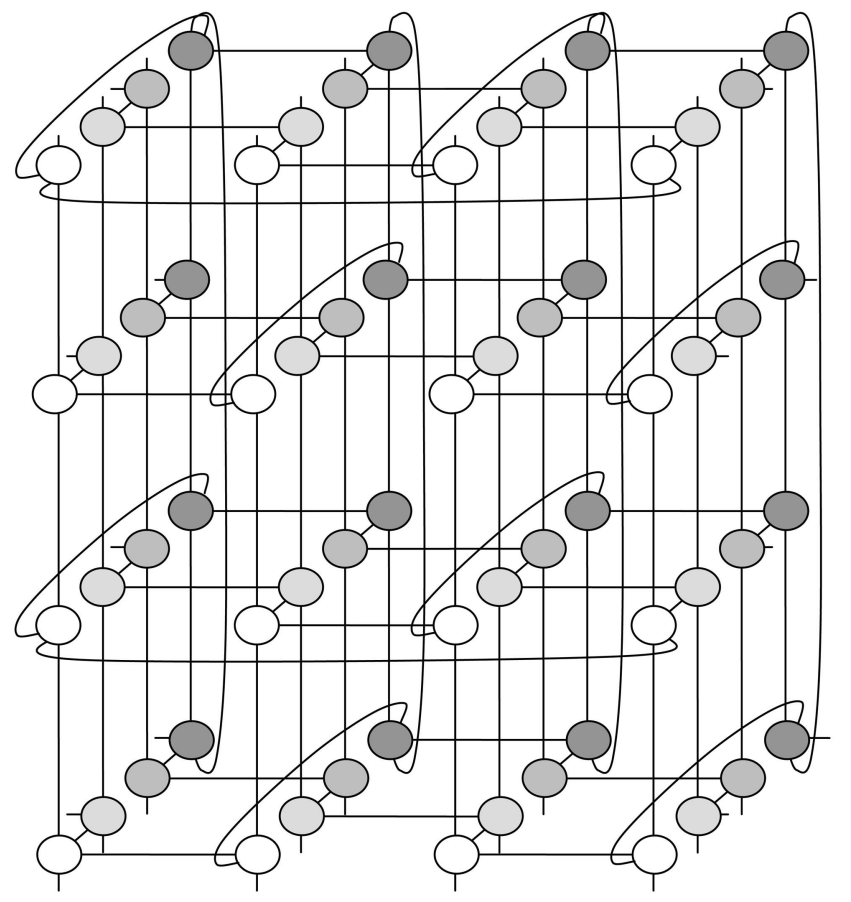

Fig. 3. A 3D diamond network; to avoid clutter, only four of the 16 vertical and two out of eight horizontal wraparound links are shown in full.

We now define a mapping $\otimes$ on $G$. For any $x, y \in G$, let

$$
x \otimes y=x+M^{f(x)} y,
$$

where $M$ is a matrix of order $q$ and $f$ is a function from $G$ to $Z_{l}$ such that $M^{f(x)}$ is well defined. That condition 1 is satisfied can be easily established: $x \otimes 1=x$ for any $x \in G$, where $t=(0,0, \ldots, 0)^{t}$ is the all-0s vector of length $q$. Similarly, condition 2 is also satisfied: Any element $x$ of $G$ has a right inverse $-M^{-f(x)} x$. In fact, letting $y=-M^{-f(x)} x$, we have:

$$
x \otimes y=x+M^{f(x)} y=x+M^{f(x)}\left(-M^{-f(x)} x\right)=x-I_{q} x=4 .
$$


Hence, to define a group operator $\otimes$ on the set $G$ by means of (4), it suffices to ensure that the associative law 3 holds for the operator $\otimes$. We have:

$$
(x \otimes y) \otimes z=\left(x+M^{f(x)} y\right) \otimes z=x+M^{f(x)} y+M^{f(x \otimes y)} z .
$$

On the other hand, we have:

$$
\begin{aligned}
x \otimes(y \otimes z) & =x+M^{f(x)}(y \otimes z)=x+M^{f(x)}\left(y+M^{f(y)} z\right) \\
& =x+M^{f(x)} y+M^{f(x)+f(y)} z .
\end{aligned}
$$

Therefore, the associative law is satisfied for the operator $\otimes$ if and only if, for any $x, y, z \in G$,

$$
M^{f(x \otimes y)} z=M^{f(x)+f(y)} z,
$$

or, if and only if, for any $x, y \in G$,

$$
M^{f(x \otimes y)}=M^{f(x)+f(y)} .
$$

Let $M$ and $f$ be as above. We can now state our main theorem.

Theorem 1. If (4) and (5) hold for any $x, y \in G$, then $(G, \otimes)$ is a group.

We give some corollaries to Theorem 1 in the following: Since $M^{f(x)}$ is well defined, $\phi: x \rightarrow M^{f(x)}$ is a mapping from the set $G$ to the set $\left\{M^{f(x)} \mid x \in G\right\}$. Letting $H=(G, \otimes)$, we have the following corollary:

Corollary 1. The set $\left\{M^{f(x)} \mid x \in H\right\}$ is a group on matrix multiplication and the mapping $\phi$ is a homomorphism from the group $H$ to the group $\left\{M^{f(x)} \mid x \in H\right\}$.

Proof. We have $\phi(x)=M^{f(x)}, \phi(y)=M^{f(y)}$, and, so, $\phi(x \otimes y)=M^{f(x \otimes y)}=M^{f(x)+f(y)}=\phi(x) \phi(y)$.

\section{Corollary 2.}

1. $x^{-1}=-M^{-f(x)} x$ for $x \in H$.

2. $M^{f\left(x^{-1}\right)}=M^{-f(x)}$ for $x \in H$.

3. $y^{-1} \otimes x \otimes y=y^{-1}+M^{-f(y)} x+M^{f(x)-f(y)} y$ for $x, y \in H$.

Proof.

1. We have $t=x \otimes x^{-1}=x+M^{f(x)} x^{-1}$ and, so, $x^{-1}=-M^{-f(x)} x$.

2. We have $y=1 \otimes y=4+M^{f(1)} y=M^{f(1)} y$ and, so, $M^{f(1)}$ is the identity matrix. Thus:

$$
\begin{aligned}
M^{f(x)+f\left(x^{-1}\right)}= & M^{f\left(x \otimes x^{-1}\right)}=M^{f(1)}, \\
& \text { and, so, } M^{f\left(x^{-1}\right)}=M^{-f(x)} .
\end{aligned}
$$

3. By statement 2 just proven, we have:

$$
\begin{aligned}
y^{-1} \otimes x \otimes y & =y^{-1}+M^{f\left(y^{-1}\right)}\left(x+M^{f(x)} y\right) \\
& =y^{-1}+M^{f\left(y^{-1}\right)} x+M^{f\left(y^{-1}\right)+f(x)} y \\
& =y^{-1}+M^{-f(y)} x+M^{f(x)-f(y)} y .
\end{aligned}
$$

Corollary 3. Let $\Gamma=\operatorname{Cay}(G, S)$ and $\Sigma=\operatorname{Cay}(H, T)$ be as introduced earlier in this section. Then, $\Sigma$ is a pruned network obtained from $\Gamma$ if and only if $M^{f(x)} T \subseteq S$ for any $x \in G$ as a set.

Proof. $\Sigma$ is a pruned network obtained from $\Gamma$ if and only if, for any $z \in T$, there is some $s \in S$ such that $x \otimes z=x+s$. But, $x \otimes z=x+M^{f(x)} z$. Thus, $\Sigma$ is a pruned network obtained from $\Gamma$ if and only if $M^{f(x)} T \subseteq S$ for any $x \in G$ as a set.

Remark 1. By Corollary 3, it can be verified that cubeconnected cycles, honeycomb, and diamond networks are obtainable from corresponding torus networks via pruning.

Remark 2. By Theorem 1 and Corollary 3, we may construct a series of pruned networks from given torus networks. Thus, we get a systematic and unified method of constructing new and useful interconnection networks.

\section{General Results for Constructing New NETWORKS}

By suitably selecting the matrix $M$ and the function $f$ of Section 3, we may construct a series of new interconnection networks according to Theorem 1 . We first give a useful definition.

Definition 1. A matrix $M$ of order $q$ is a quasipermutation matrix if only one element of each row and each column of $M$ is 1 or -1 and all other elements are zero. Thus, in terms of zero and nonzero elements, a quasipermutation matrix $M$ of order $q$ has the appearance as a permutation matrix, which is derived by permuting the rows of $I_{q}$ (the identity matrix of order q).

We consider two classes of interconnection networks in the following.

For the first class of interconnection networks, we let $G=Z_{l}^{q} \times Z_{k}, M=\left[\begin{array}{cc}Q & 0 \\ 0 & 1\end{array}\right]$, where $Q$ is a quasipermutation matrix of order $q$ such that $Q^{k}=I_{q}$, the identity matrix of order $q$. Let $f(x)=x_{q+1}$. Then, $f$ is a function from $G$ to $Z_{k}$. Defining $a \otimes b=a+M^{a_{q+1}} b$, we have:

$$
M^{f(x \otimes y)}=M^{x_{q+1}+y_{q+1}}=M^{f(x)+f(y)} .
$$

By Theorem $1, \otimes$ is a group operator on the set $G$. Let $S$ and $T$ be as above. Then, $M S \subseteq S$ and so $M^{f(x)} T \subseteq S$ for any $x \in G$. By Corollary 3, the Cayley graph $\Sigma=\operatorname{Cay}(H, T)$ is a pruned network obtained from the Cayley graph $\Gamma=\operatorname{Cay}(G, S)$. It is clear that the cube-connected cycles $C C C_{q}$ belongs to this class of interconnection networks.

For the second class of interconnection networks, we let $G=Z_{k_{1}} \times Z_{k_{2}} \times \ldots \times Z_{k_{q+1}}$, where $k_{i}$ is an even integer for $1 \leq i \leq q+1$. Assume that $M$ is a quasipermutation matrix of order $q+1$ and that $M^{2}=I_{q+1}$, the identity matrix of order $q+1$. Let $f$ be a function from $G$ to $Z_{2}$ such that $f(x)=x_{1}+x_{2}+\cdots+x_{q+1}$ for any $x \in G$. Define the operator $\otimes$ by $x \otimes y=x+M^{f(x)} y$, where $x, y \in G$. Then, $M^{f(x \otimes y)}=M^{f(x)+f(y)}$ and, so, $\otimes$ is a group operator on $G$. Let $S, T$, and $H$ be as above and $\Sigma=\operatorname{Cay}(H, T)$. It is possible that $\Sigma$ is not a pruned network obtained from 


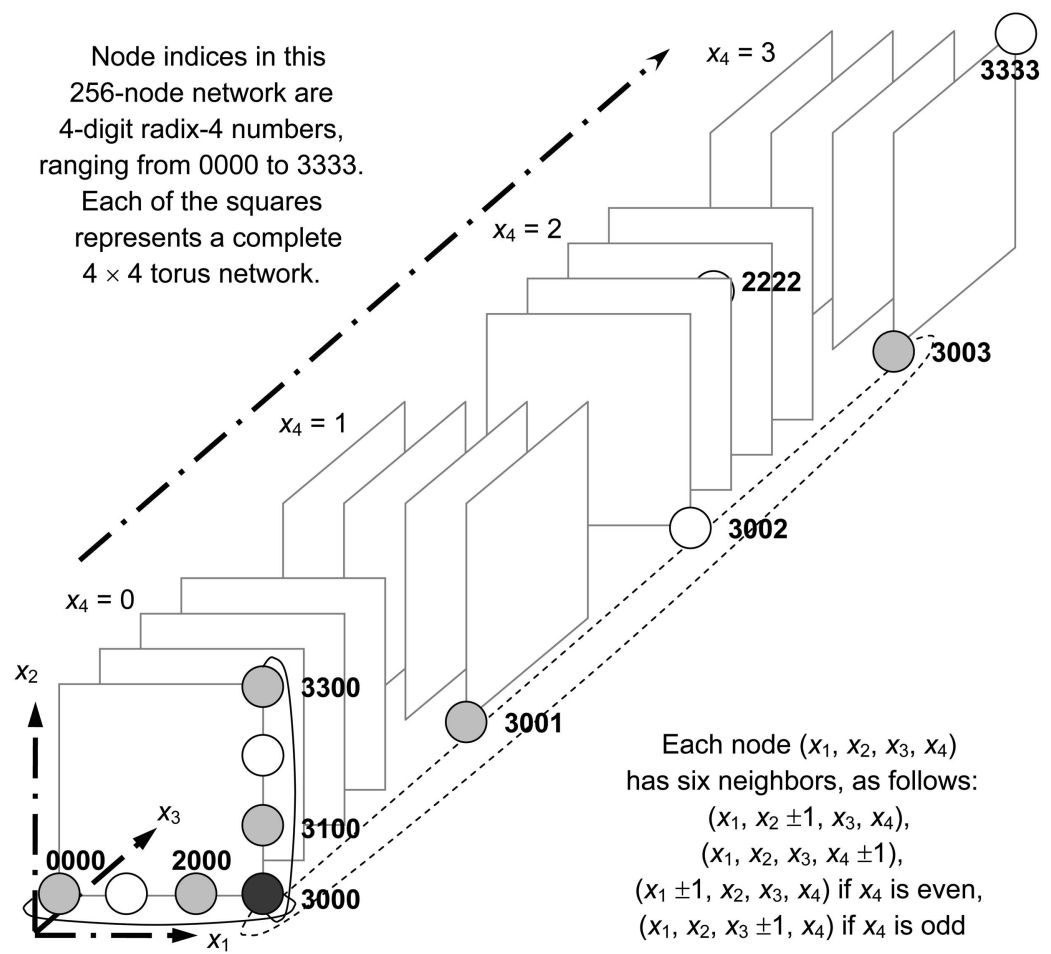

Fig. 4. The 4D pruned network of Example 4. Only one of the 64 cycles of length 4 along the $x_{4}$ dimension is shown (broken lines).

the graph $\Gamma=\operatorname{Cay}(G, S)$ because it may be the case that $M^{f(x)} T \not \subset S$ for some $x \in G$. But, if all $k_{i}(1 \leq i \leq q+1)$ are the same, then $M^{f(x)} T \subseteq S$ holds for any $x \in G$. Hence, $\Sigma$ is a pruned network obtained from $\Gamma$ by Corollary 3. On the other hand, when

$$
M=\left[\begin{array}{cccc}
-1 & \cdots & 0 & 0 \\
\vdots & \ddots & \vdots & \vdots \\
0 & \cdots & -1 & 0 \\
0 & \cdots & 0 & 1
\end{array}\right]
$$

we have $M^{f(x)} T \subseteq S$ for any $x \in G$. Thus, the graph $\Sigma$ is a pruned network obtained from $\Gamma$. It is evident that honeycomb and diamond networks belong to this class of networks [9].

To show the power of our method, beyond serving as a unifying model of interconnection networks that are already known, we present an example of a new network that has not been previously proposed or studied. Note that, owing to the intuitive appeal as well as simpler visualization and analysis for 3D networks, much of the published studies in this area have been focused on pruned versions of 3D networks.

Example 4. We can extend the $T_{1}$ network of [5] to four dimensions, obtaining a pruned interconnection network that has some advantages of $4 \mathrm{D}$ networks (such as lower diameter) and the implementation ease of lower-dimensional networks, while maintaining the allimportant property of node symmetry. Let $G=Z_{k}^{4}$ and $\Gamma=\operatorname{Cay}(G, S)$. For any $x \in G$, we write $x=\left(x_{1}, x_{2}, x_{3}\right.$, $\left.x_{4}\right)^{t}$ and define $f(x)=x_{4}$. For $x, y \in G$, define the new group operator $\otimes$ based on the $4 \times 4$ permutation matrix $M$ as follows:

$$
x \otimes y=x+\left[\begin{array}{llll}
0 & 0 & 1 & 0 \\
0 & 1 & 0 & 0 \\
1 & 0 & 0 & 0 \\
0 & 0 & 0 & 1
\end{array}\right]^{x_{4}} y .
$$

We have $H=\langle a, b, c\rangle\langle d\rangle$, where $a=(1,0,0,0)^{t}, \quad b=$ $(0,1,0,0)^{t}, c=(0,0,1,0)^{t}, d=(0,0,0,1)^{t}$, with $a, b, c$, and $d$ satisfying the following relations:

$$
\begin{aligned}
& a^{k}=b^{k}=c^{k}=d^{k}=4 ; a b=b a ; a c=c a ; b c=c b \\
& d^{-1} a d=c ; d^{-1} c d=a ; d^{-1} b d=b .
\end{aligned}
$$

Letting $T=\left\{a^{ \pm 1}, b^{ \pm 1}, d^{ \pm 1}\right\}$ and $\Sigma=\operatorname{Cay}(H, T)$, we observe that $\Sigma$ is a pruned network obtained from $\Gamma$. Fig. 4 depicts the structure of the network $\Sigma$, where the neighbors of $x=$ $\left(x_{1}, x_{2}, x_{3}, x_{4}\right)^{t}$ are as follows: $x \otimes b^{ \pm 1}=x \pm b=\left(x_{1}, x_{2}\right.$ $\left.\pm 1, x_{3}, x_{4}\right)^{t}, x \otimes d^{ \pm 1}=x \pm d=\left(x_{1}, x_{2}, x_{3}, x_{4} \pm 1\right)^{t}, x \otimes a^{ \pm 1}$ $=x \pm a=\left(x_{1} \pm 1, x_{2}, x_{3}, x_{4}\right)^{t}$ if $x_{4}$ is even, and $x \otimes a^{ \pm 1}=$ $x \pm c=\left(x_{1}, x_{2}, x_{3} \pm 1, x_{4}\right)^{t}$ if $x_{4}$ is odd.

The diameter of the network $\Sigma$ of Example 4 is $2 k$, except in the case of $k=2$, when it is 5 . This is easily proven. For $k=2$, we have $\operatorname{dist}(4, v) \leq 5$, for all $v \in H$, and $\operatorname{dist}(1, a b c)=\operatorname{dist}(1, a b d a d)=5$. For $k \geq 4$, we note that no node is at a greater distance from node $(0,0,0,0)^{t}$ and that node $u=a^{k / 2} b^{k / 2} c^{k / 2} d^{k / 2}=a^{k / 2} b^{k / 2} d a^{k / 2} d^{k / 2-1}$ is at distance $2 k$. For example, a diametral path of length 8 in the network of Fig. 4, beginning at node 0000, is as follows: $0000 \rightarrow 1000 \rightarrow 2000 \rightarrow 2100 \rightarrow 2200 \rightarrow 2201 \rightarrow$ $2211 \rightarrow 2221 \rightarrow 2222$ (the last node is also depicted in Fig. 4). Note that this network resembles a 4D network in terms of diameter and average internode distance, but has an implementation cost (node degree, VLSI layout complexity) that is close to those of $3 \mathrm{D}$ networks. This 
is a recurring theme for interconnection networks that are obtained by judicious pruning of more complex networks (that have higher dimensions as well as more links) and constitutes the main motivation for our interest in a systematic study of network pruning schemes.

\section{New Results for 3D InTERCONNECTION NETWORKS}

In this section, we consider certain 3D interconnection networks in detail. Let $G=Z_{k}^{3}$ as in Example 3, where $k$ is an even integer. For $x, y \in G$, define a new group operator $\otimes$ as $x \otimes y=x+M^{f(x)} y$, where the matrix $M$ is one of the three matrices below:
(1) $M=\left[\begin{array}{ccc}0 & -1 & 0 \\ -1 & 0 & 0 \\ 0 & 0 & 1\end{array}\right]$,
(2) $M=\left[\begin{array}{lll}0 & 1 & 0 \\ 1 & 0 & 0 \\ 0 & 0 & 1\end{array}\right]$,
(3) $M=\left[\begin{array}{ccc}-1 & 0 & 0 \\ 0 & -1 & 0 \\ 0 & 0 & 1\end{array}\right]$.

The function $f$ is chosen in two possible ways:
(A) $f(x)=x_{3}$ and
(B) $f(x)=x_{1}+x_{2}+x_{3}$.

In the following discussion, we omit the operator $\otimes$ in expressions. We consider the six possible cases $1 \mathrm{~A}$ (case 1 of the matrix $M$ and case A of the function $f$ ), 1B, 2A, 2B, 3A, and $3 \mathrm{~B}$ in turn.

For case $1 \mathrm{~A}$, we have $H=(\langle a\rangle \times\langle b\rangle)\langle c\rangle$, where $a=(1,0,0)^{t}, \quad b=(0,1,0)^{t}, c=(0,0,1)^{t}$ and $a, b$, and $c$ satisfy the following relations:

$$
a^{k}=b^{k}=c^{k}=1 ; \quad a b=b a ; \quad c^{-1} a c=b^{-1} ; \quad c^{-1} b c=a^{-1} .
$$

Let $T=\left\{a^{ \pm 1}, c^{ \pm 1}\right\}$ and $\Sigma=\operatorname{Cay}(H, T)$. Then, $\Sigma$ is the $T_{1}$ network of [5].

For case $1 \mathrm{~B}$, we have $H=\langle a, b\rangle\langle c\rangle$, where $a=(1,0,0)^{t}$, $b=(0,-1,0)^{t}, c=(0,0,1)^{t}$, and $a, b$, and $c$ satisfy the following relations:

$$
\begin{aligned}
& a^{2 k}=b^{2 k}=c^{k}=4 ; \quad c^{-1} a c=b ; \quad c^{-1} b c=a ; \\
& (a b)^{k / 2}=(b a)^{k / 2}=1 .
\end{aligned}
$$

Let $T=\left\{a^{ \pm 1}, c^{ \pm 1}\right\}$ and $\Sigma=\operatorname{Cay}(H, T)$. Then, $\Sigma$ is the $T_{2}$ network of [5].

For cases $2 \mathrm{~A}$ and $2 \mathrm{~B}$, it is easily verified that the corresponding pruned network $\Sigma$ is isomorphic to the $T_{1}$ and $T_{2}$ networks of [5], respectively.

For case $3 \mathrm{~A}$, we have $H=(\langle a\rangle \times\langle b\rangle)\langle c\rangle$, where $a=(1,0,0)^{t}, b=(0,1,0)^{t}, c=(0,0,1)^{t}$, and $a, b$, and $c$ satisfy the following relations:

$$
a^{k}=b^{k}=c^{k}=1 ; \quad a b=b a ; \quad c^{-1} a c=a^{-1} ; \quad c^{-1} b c=b^{-1} .
$$

Let $T=\left\{a^{ \pm 1}, b^{ \pm 1}, c^{ \pm 1}\right\}$ and $\Sigma=\operatorname{Cay}(H, T)$. Then, $\Sigma$ is isomorphic to the $3 \mathrm{D}$ torus.

For case $3 \mathrm{~B}$, we have $H=\langle d, a, e, b\rangle\langle c\rangle$, where $a=(1,0,0)^{t}, b=(0,1,0)^{t}, c=(0,0,1)^{t}$ and $a, b, c, d$, and $e$ satisfy the following relations:

$$
\begin{aligned}
& a^{2}=b^{2}=c^{k}=d^{k / 2}=e^{k / 2}=4 ; a d a=d^{-1} ; c a c^{-1}=d^{-1} a ; \\
& c d c^{-1}=d^{-1} ; b e b=e^{-1} ; c b c^{-1}=e^{-1} b ; c e c^{-1}=e^{-1} .
\end{aligned}
$$

Let $T=\{a, b, c\}$ and $\Sigma=\operatorname{Cay}(H, T)$. Then, $\Sigma$ is the 3D diamond network [9].

We next consider the structure of the group $H$. It is clear that $\langle d, a\rangle=\langle d\rangle\langle a\rangle$ is a semidirect product of $\langle d\rangle$ by $\langle a\rangle$ since $a d a=d^{-1}$. Similarly, $\langle e, b\rangle=\langle e\rangle\langle b\rangle$ is a semidirect product of $\langle e\rangle$ by $\langle b\rangle$. Hence, $|\langle d, a\rangle|=|\langle e, b\rangle|=k$, that is, their order is $k$. Because $\langle d, a\rangle \cap\langle e, b\rangle=1$, the order of $\langle d, a\rangle\langle e, b\rangle$ is $k^{2}$. By the relations among $a, b, c, d$, and $e, H=\langle d, a, e, b\rangle\langle c\rangle$ is a semidirect product of $\langle d, a, e, b\rangle$ by $\langle c\rangle$. Thus, we obtain that the order of $\langle d, a, e, b\rangle$ is $k^{2}$. Therefore, $\langle d, a, e, b\rangle=\langle d, a\rangle\langle e, b\rangle$ and, so, $H=(\langle d\rangle\langle a\rangle)(\langle e\rangle\langle b\rangle)\langle c\rangle$. It is easily verified that $d e^{-1}=$ $a b a b$ and $d e=e d$.

We illustrate the method above by using it to compute the diameter of the 3D diamond network.

Theorem 2. Let $\Sigma$ be as above. Then, the diameter of $\Sigma$ is $3 k / 2$, where $k$ is an even integer.

Proof. Let the diameter of the diamond network $\Sigma$ be $\operatorname{Diam}(\Sigma)$. It is easily verified that $c^{2}$ is commutative with any elements of $H$. For any $g, h \in H$, the distance between $g$ and $h$ is denoted $\operatorname{dist}(g, h)$. It is clear that $\operatorname{dist}(1, a b c)=3$ and, so, $\operatorname{Diam}(\Sigma)=3$ when $k=2$. Similarly, we can prove that $\operatorname{Diam}(\Sigma)=6$ when $k=4$ because $d e=a b a b$ and $\operatorname{dist}\left(1, \operatorname{dec}^{2}\right)=\operatorname{dist}\left(1, a b a b c^{2}\right)=6$. Now, let $k$ be any even integer. We consider two cases, depending on whether 4 divides $k$.

If $k$ is a multiple of 4 , then we have:

$$
\operatorname{dist}\left(1,(d e)^{k / 4} c^{k / 2}\right)=\operatorname{dist}\left(1,(a b a b)^{k / 4} c^{k / 2}\right)=3 k / 2 .
$$

On the other hand, we can prove that, for any $g \in H$, $\operatorname{dist}(1, g) \leq 3 k / 2$ because $c^{2}$ is commutative with any elements of $H$.

If $k$ is not a multiple of 4 , then we have:

$$
\begin{aligned}
& \operatorname{dist}\left(1,\left(d e^{-1}\right)^{(k-2) / 4} a b c^{k / 2}\right)=\operatorname{dist}\left(1,(a b a b)^{(k-2) / 4} a b c^{k / 2}\right) \\
& =3 k / 2 .
\end{aligned}
$$

In a manner similar to that of the first case, we can prove that, for any $g \in H$, $\operatorname{dist}(t, g) \leq 3 k / 2$. The two cases together establish the result that $\operatorname{Diam}(\Sigma)=3 k / 2$ for any even $k$.

Remark 3. In [9], the authors showed that the diameter of the pruned 3D torus [10] is $3 k / 2$. Theorem 2 establishes that the pruned 3D torus, which has the same node and wiring complexity as the $3 \mathrm{D}$ diamond network, is also identical to the latter in terms of diameter.

\section{ConClusion}

In this paper, we have provided a general group construction from a commutative group. It generalizes previously proposed pruning schemes and provides a systematic and unified framework of constructing interconnection networks. As an application of our method, we also derived some new results on pruned networks, such as honeycomb and diamond networks. Because of the generality of these results, which can be viewed as allowing the synthesis of alternative, more economical, interconnection networks by 
reducing the number of dimensions and/or link density of existing networks via mapping and pruning, we expect that they will find many more applications.

Many problems remain to be studied. We are currently investigating the applications of our method to problems related to routing and average internode distance in certain subgraphs and coset graphs of diamond and infinite honeycomb networks. These results, along with potential applications in the following areas, will be reported in the future:

- Load balancing and congestion control.

- Scheduling and resource allocation.

- Fault tolerance and graceful degradation.

These constitute important practical problems in the design, evaluation, and efficient operation of parallel and distributed computer systems. Another interesting open problem is the extent to which Cayley graphs that are incomplete $k$-ary $n$-cubes can be derived within our framework with appropriate choices of $M, f(x)$, and $T$. In other words, does there exist such a Cayley graph that does not belong to the class of networks derived from this formulation?

\section{ACKNOWLEDGMENTS}

The authors thank an anonymous referee who brought an error in Theorem 2 to their attention and who suggested some improvements and problems for further investigation. Wenjun Xiao's research was supported by the Natural Science Foundation of China and Guangdong Province.

\section{REFERENCES}

[1] S.B. Akers and B. Krishnamurthy, "A Group Theoretic Model for Symmetric Interconnection Networks," IEEE Trans. Computers, vol. 38, no. 4, pp. 555-566, Apr. 1989.

[2] F. Annexstein, M. Baumslag, and A.L. Rosenberg, "Group Action Graphs and Parallel Architectures," SIAM J. Computing, vol. 19, no. 3, pp. 544-569, June 1990.

[3] N. Biggs, Algebraic Graph Theory, second ed. Cambridge Univ. Press, 1993.

[4] M. Heydemann, "Cayley Graphs and Interconnection Networks," Graph Symmetry: Algebraic Methods and Applications, G. Hahn and G. Sabidussi, eds., pp. 167-224, Kluwer Academic, 1997.

[5] D.M. Kwai and B. Parhami, "Pruned Three-Dimensional Toroidal Networks," Information Processing Letters, vol. 68, no. 4, pp. 179183, Nov. 1998.

[6] F.T. Leighton, Introduction to Parallel Algorithms and Architectures: Arrays, Trees, Hypercubes. Morgan Kaufmann, 1992.

[7] F.G. Nocetti, I. Stojmenovic, and J. Zhang, "Addressing and Routing in Hexagonal Networks with Applications for Tracking Mobile Users and Connection Rerouting in Cellular Networks," IEEE Trans. Parallel and Distributed Systems, vol. 13, no. 9, pp. 963971, Sept. 2002.

[8] B. Parhami, Introduction to Parallel Processing: Algorithms and Architectures. Plenum Press, 1999.

[9] B. Parhami and D.M. Kwai, "A Unified Formulation of Honeycomb and Diamond Networks," IEEE Trans. Parallel and Distributed Systems, vol. 12, no. 1, pp. 74-80, Jan. 2001.

[10] B. Parhami and D.M. Kwai, "Incomplete $k$-Ary $n$-Cube and Its Derivatives," J. Parallel and Distributed Systems, vol. 64, no. 2, pp. 183-190, Feb. 2004.

[11] I. Stojmenovic, "Honeycomb Networks: Topological Properties and Communication Algorithms," IEEE Trans. Parallel and Distributed Systems, vol. 8, no. 10, pp. 1036-1042, Oct. 1997.

[12] W. Xiao and B. Parhami, "Some Mathematical Properties of Cayley Digraphs with Applications to Interconnection Network Design," Int'l J. Computer Math., vol. 82, no. 5, pp. 521-528, May 2005.

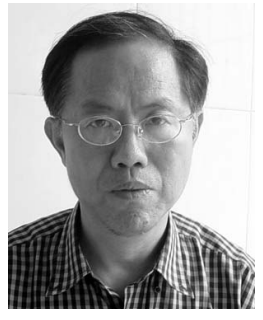

Wenjun Xiao received the $\mathrm{PhD}$ degree in mathematics from Sichuan University, People's Republic of China, in 1989. Currently, he is a professor in the School of Computer Science and Engineering, South China University of Technology, Guangzhou, People's Republic of China. His research interests include discrete mathematics, parallel and distributed computing, complex networks, and software architecture. $\mathrm{He}$ has published more than 50 papers in journals and conferences on these topics since 1985.

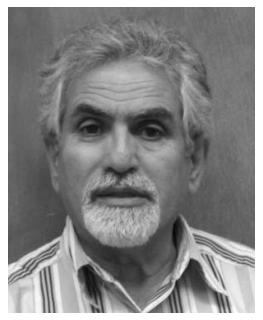

Behrooz Parhami received the $\mathrm{PhD}$ degree in computer science from the University of California, Los Angeles, in 1973. Presently, he is a professor in the Department of Electrical and Computer Engineering, University of California, Santa Barbara. His research deals with parallel architectures and algorithms, computer arithmetic, and reliable computing. In his previous position with Sharif University of Technology in Tehran, Iran (1974-1988), he was also involved in the areas of educational planning, curriculum development, standardization efforts, technology transfer, and various editorial responsibilities, including a five-year term as editor of Computer Report, a Farsilanguage computing periodical. Dr. Parhami's technical publications include more than 220 papers in journals and international conferences, a Farsi-language textbook, and an English/Farsi glossary of computing terms. Among his latest publications are two graduate-level textbooks on parallel processing (Plenum, 1999) and computer arithmetic (Oxford, 2000), and an introductory textbook on computer architecture (Oxford, 2005). Dr. Parhami is a fellow the IEEE and the IEEE Computer Society, a chartered fellow of the British Computer Society, a member of the ACM, and a distinguished member of the Informatics Society of Iran, for which he served as a founding member and president from 1979-1984. $\mathrm{He}$ also served as chairman of the IEEE Iran Section (1977-1986) and received the IEEE Centennial Medal in 1984.

\footnotetext{
$\triangleright$ For more information on this or any other computing topic,
} please visit our Digital Library at www.computer.org/publications/dlib. 